\title{
On the use of Hadamard expansions in hyperasymptotic evaluation: differential equations of hypergeometric type
}

\author{
D. Kaminski \\ Department of Mathematics and Computer Science, \\ University of Lethbridge, Lethbridge, Alberta T1K 3M4, Canada
}

R. B. Paris

Division of Mathematical Sciences, University of Abertay Dundee, Dundee DD1 1HG, UK

(MS received 9 May 2003; accepted 10 September 2003)

\begin{abstract}
We describe how a modification of a common technique for developing asymptotic expansions of solutions of linear differential equations can be used to derive Hadamard expansions of solutions of differential equations. Hadamard expansions are convergent series that share some of the features of hyperasymptotic expansions, particularly that of having exponentially small remainders when truncated, and, as a consequence, provide a useful computational tool for evaluating special functions. The methods we discuss can be applied to linear differential equations of hypergeometric type and may have wider applicability.
\end{abstract}

\section{Introduction}

A Hadamard expansion is one of the special form

$$
f(z)=\sum_{n=0}^{\infty} \mathrm{e}^{-\lambda_{n} z} S_{n}(z)
$$

where $S_{n}$, in turn, is a series of the form

$$
S_{n}(z)=\sum_{k=0}^{\infty} \frac{a_{n k}}{\left(\rho_{n} z\right)^{\mu_{n}+k}} P\left(\mu_{n}+k, \rho_{n} z\right) .
$$

The function $P$ appearing in the sum defining $S_{n}(z)$ is the normalized incomplete gamma function given by

$$
P(a, z)=\frac{1}{\Gamma(a)} \int_{0}^{z} \mathrm{e}^{-t} t^{a-1} \mathrm{~d} t, \quad|\arg z|<\pi, \quad \operatorname{Re}(a)>0,
$$

and all other terms in the sum (1.2), $\mu_{n}, \rho_{n}$ and $a_{n k}$, are constants. We will refer to the index $n$ as the level of the expansion, and the parameter $\lambda_{n}$ appearing in the exponential factor $\mathrm{e}^{-\lambda_{n} z}$ will be assumed to have argument restricted in such a way that $\operatorname{Re}\left(\lambda_{n} z\right) \geqslant 0$. We also assume that $\operatorname{Re}\left(\lambda_{n} z\right) \leqslant \operatorname{Re}\left(\lambda_{n+1} z\right)$ for $n=0,1,2, \ldots$ 


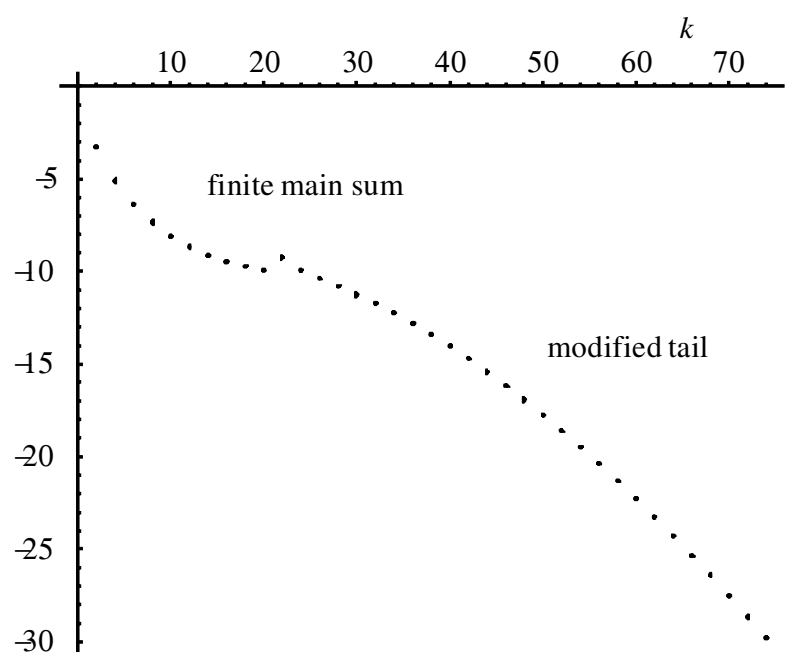

Figure 1. Magnitudes of the terms (plotted on a $\log _{10}$ scale) in the modified Hadamard expansion of $I_{\nu}(x)$ against ordinal number $k$ when $x=10, \nu=\frac{1}{4}$ and the truncation index $M=22$.

We mention here, for later reference, that the incomplete gamma function has the asymptotic behaviour

$$
P(a, z)=\frac{z^{a} \mathrm{e}^{-z}}{\Gamma(1+a)}{ }_{1} F_{1}(1 ; 1+a ; z) \sim \begin{cases}1-\frac{z^{a-1} \mathrm{e}^{-z}}{\Gamma(a)}, & z \rightarrow+\infty, \\ \frac{z^{a} \mathrm{e}^{-z}}{\Gamma(1+a)}, & a \rightarrow+\infty .\end{cases}
$$

Hadamard expansions have been the subject of prior work by one of the authors (see [5-7]), and the interested reader will find many features of these expansions described at length. For the benefit of the reader new to Hadamard expansions, we point out a few salient features of these types of representations.

First, unlike most expansions encountered in asymptotics, Hadamard expansions are usually absolutely convergent, and the asymptotic scale for the expansions at level $n,\left\{P\left(\mu_{n}+k, \rho_{n} z\right) /\left(\rho_{n} z\right)^{\mu_{n}+k}\right\}$, is exponentially negligible in the scale at the previous level $n-1$, due to the exponentially small factors $\mathrm{e}^{-\lambda_{n} z}$ associated with each level. Secondly, because of the asymptotic behaviour of the incomplete gamma function in (1.4a), the Hadamard expansion at level zero, for large $z$, can be seen to contain (to leading order) the usual Poincaré asymptotic expansion. This is easily understood in the example of the modified Bessel function $I_{\nu}(x)$ (see [5] and [8, p. 204]), which has a particularly simple Hadamard expansion, valid when $\operatorname{Re}(\nu)>-\frac{1}{2}$, consisting of a single level given by

$$
I_{\nu}(x)=\frac{\mathrm{e}^{x}}{\sqrt{2 \pi x}} \sum_{k=0}^{\infty} \frac{a_{k}(\nu)}{(2 x)^{k}} P\left(\frac{1}{2}+\nu+k, 2 x\right),
$$

where $a_{k}(\nu)=\left(\frac{1}{2}+\nu\right)_{k}\left(\frac{1}{2}-\nu\right)_{k} / k !$ and $(a)_{k} \equiv \Gamma(a+k) / \Gamma(a)$ is Pochhammer's symbol. We point out that a more general form of the Hadamard expansion for 
$I_{\nu}(x)$ has been discussed in [3]. If the factor $P\left(\frac{1}{2}+\nu+k, 2 x\right)$ in this expansion is formally replaced by unity, the usual Poincaré expansion for $I_{\nu}(x)$ is obtained valid for $x \rightarrow+\infty$. It follows then that Hadamard expansions have terms that initially undergo a decay in magnitude comparable to ordinary asymptotic expansions, but, in marked contrast to most asymptotic expansions that eventually diverge to infinity, the terms ultimately go over into a gradual decay of algebraic order.

Owing to this gradual decay, Hadamard expansions in their untransformed state are ill suited as an effective computational tool. However, if we truncate each Hadamard series $S_{n}(z)$ in (1.1) at an appropriate point, thereby producing a finite main sum and a tail at each level, the tail resulting from this truncation can be transformed into one that exhibits rapid decay-often comparable with that of the initial asymptotic-like phase thereby permitting the truncated Hadamard series and associated tails to be used as an efficient computational tool. This truncation process results in what we shall call a modified Hadamard expansion. It is important to stress that the modified tails are still absolutely convergent and that no approximation has been introduced in the process.

We explain this procedure in the comparatively simple setting of the expansion for $I_{\nu}(x)$ in $(1.5)$, which we write as

$$
I_{\nu}(x)=\frac{\mathrm{e}^{x}}{\sqrt{2 \pi x}}\left\{\sum_{k=0}^{M-1} \frac{a_{k}(\nu)}{(2 x)^{k}} P\left(\frac{1}{2}+\nu+k, 2 x\right)+T(M ; 2 x)\right\},
$$

where $M$ is the truncation index and the tail $T(M ; 2 x)$ is the series

$$
T(M ; 2 x)=\sum_{k=M}^{\infty} \frac{a_{k}(\nu)}{(2 x)^{k}} P\left(\frac{1}{2}+\nu+k, 2 x\right) .
$$

It is shown in [5] that $T(M ; 2 x)$, after representing the incomplete gamma function in terms of ${ }_{1} F_{1}$ (see $(1.4)$ ), followed by expansion of ${ }_{1} F_{1}$ in terms of its Maclaurin series and a reversal in the order of summation, can be rendered in the form

$$
T(M ; 2 x)=\mathrm{e}^{-2 x} \sum_{r=0}^{\infty} \sigma_{r}(M)\left(\frac{2 x}{M}\right)^{\nu+r+1 / 2},
$$

where the coefficients $\sigma_{r}$ have an explicit representation in terms of a ${ }_{2} F_{1}$ function. Provided $M$ is chosen to satisfy $M \geqslant\lfloor 2 x\rfloor+1$, the terms in the tail re-expressed this way are found to undergo a rapid decay as the index $r$ increases, as is illustrated in figure 1. We also remark that the rapidly convergent series has been displayed with an exponentially small factor $\mathrm{e}^{-2 x}$, which gives an indication of the level at which the tail contributes to the Hadamard expansion. It is for these reasons that the modified Hadamard expansion provides a powerful mechanism for computing $I_{\nu}(x)$ to high precision.

This rendering of tails in Hadamard expansions into rapidly convergent forms is a crucial difference in the usual treatment accorded to remainders in asymptotic expansions. The reader interested in a systematic account of the theory of Hadamard expansions is directed to the previously mentioned works, which include the development and analysis of the tails resulting from the truncation of an expansion. 
The paper is arranged as follows. The technique of developing Hadamard expansions from differential equations is broadly described in the next section and subsequently refined and applied to Kummer's equation for the confluent hypergeometric functions. A detailed account of the tails resulting from truncation is given, without any appeal to the results for integrals found in [5,6]. A numerical illustration of the results for Kummer's equation is included to demonstrate the accuracy that can be achieved with this procedure. The paper concludes with some further discussion of the problem of determining Hadamard expansions from other types of differential equations.

\section{Expansions from differential equations}

We follow the well-known technique used in forming Poincaré-type expansions for large $z$ of solutions to linear second-order differential equations, as detailed in [4, $\S 7.1]$, and consider the equation

$$
w^{\prime \prime}(z)+f(z) w^{\prime}(z)+g(z) w(z)=0,
$$

with expansions for the coefficient functions

$$
f(z) \sim \sum_{s=0}^{\infty} \frac{f_{s}}{z^{s}} \text { and } g(z) \sim \sum_{s=0}^{\infty} \frac{g_{s}}{z^{s}},
$$

where not all of the coefficients $f_{0}, g_{0}$ and $g_{1}$ vanish. Then the method proceeds by formally substituting a trial expansion

$$
w(z) \sim \mathrm{e}^{\lambda z} z^{\mu} \sum_{s=0}^{\infty} \frac{a_{s}}{z^{s}}
$$

into the differential equation with the series expansions for $f(z)$ and $g(z)$, and determining the parameters $\lambda, \mu$ and the coefficients $a_{s}$ from recurrence relations that result. ${ }^{1}$ The success of this technique owes much to several properties of asymptotic power series. First, any reciprocal power series, when subjected to a differentiation, remains a reciprocal power series. Furthermore, the product and sum of reciprocal power series are, again, reciprocal power series. In replacing a reciprocal power series by a Hadamard expansion, however, we lose most of these attractive properties, which complicates the process of forming a Hadamard expansion counterpart to (2.2). Nevertheless, in a restricted category of linear second-order differential equations, we can resolve these complications.

The idea is relatively straightforward. For a differential equation (2.1), formally substitute

$$
w(z)=\sum_{n=0}^{\infty} \mathrm{e}^{-\lambda_{n} z} S_{n}(z)
$$

where each quantity $S_{n}(z)$ is given by

$$
S_{n}(z)=\sum_{k=0}^{\infty} a_{n k} Y_{n k}(z), \quad Y_{n k}(z)=\frac{P\left(\mu_{n}+k, \rho_{n} z\right)}{\left(\rho_{n} z\right)^{\mu_{n}+k}},
$$

${ }^{1}$ We have been deliberately brief with the account of the method. There are some subtleties, which are discussed in detail in [4, ch. 7], which we elect not to provide in our treatment. 
with $P$ given in (1.3). Upon substitution of (2.3) into (2.1), a sum involving the functions $Y_{n k}$ and their derivatives results. By applying some simple properties of the $Y_{n k}$ and their derivatives given in the following lemma, with judicious assignment of the parameters $\rho_{n}, \mu_{n}$ and $\lambda_{n}$, we can transform the resulting sum into two series, one involving only the functions $Y_{n k}$, and one free of the $Y_{n k}$. The series involving $Y_{n k}$ will be made to vanish through the determination of the coefficients $a_{n k}$, and the remaining series will comprise two types of contributions: one algebraic and one exponentially small.

It will emerge in the course of the computations that the algebraic and exponentially small terms will behave in similar manners regardless of the level. The exponentially small contribution at level $n$ will, through adjustment of parameters, be made to cancel the algebraic terms left at level $n+1$. In this way, the sum of all the contributions over all levels will satisfy (2.1).

Before illustrating this process, let us gather together some generally useful properties of the $Y_{n k}$.

Lemma 2.1. Let $Y_{n k}(z)$ be as defined in (2.4). Then

$$
\begin{aligned}
Y_{n k}^{\prime}(z) & =-\rho_{n}\left(\mu_{n}+k\right) Y_{n, k+1}(z), \\
0 & =z Y_{n k}^{\prime \prime}(z)+\left\{\left(\mu_{n}+k+1\right)+\rho_{n} z\right\} Y_{n k}^{\prime}(z)+\rho_{n}\left(\mu_{n}+k\right) Y_{n k}(z), \\
z Y_{n k}^{\prime}(z) & =-\left(\mu_{n}+k\right) Y_{n k}(z)+\frac{\mathrm{e}^{-\rho_{n} z}}{\Gamma\left(\mu_{n}+k\right)}, \\
z Y_{n k}(z) & =\frac{1}{\rho_{n}} Y_{n, k-1}(z)-\frac{\mathrm{e}^{-\rho_{n} z}}{\rho_{n} \Gamma\left(\mu_{n}+k\right)} .
\end{aligned}
$$

Proofs of these results follow easily from the Euler integral representation of the incomplete gamma function in (1.3). In all cases, $k$ is a non-negative integer. When $k=0$ in (2.8), we see that $Y_{n,-1}$ can be assigned a meaning (provided $\left.\mu_{n} \neq 0,-1,-2, \ldots\right)$, since, from (1.4) and (2.4),

$$
Y_{n,-1}(z)=\frac{\mathrm{e}^{-\rho_{n} z}}{\Gamma\left(\mu_{n}\right)}{ }_{1} F_{1}\left(1 ; \mu_{n} ; \rho_{n} z\right) .
$$

We remark that the second property listed in the lemma is of particular significance, as it clearly illustrates that the functions used in the Hadamard expansions we will develop are themselves of hypergeometric type.

\section{The confluent hypergeometric equation}

Kummer's differential equation for the confluent hypergeometric functions is (cf. [1, $\S 13.1 .1],[4, \S 7.9])$

$$
z w^{\prime \prime}+(b-z) w^{\prime}-a w=0,
$$

and the development of Hadamard expansions of solutions to this equation will serve as a template to follow when dealing with other differential equations. It is well known that solutions of this equation possess the leading behaviour $z^{-a}$ and $z^{a-b} \mathrm{e}^{z}$ for large $|z|$. We look for solutions of (3.1) of the form

$$
w(z)=\sum_{n=0}^{\infty} \mathrm{e}^{-\lambda_{n} z} S_{n}(z),
$$


where $S_{n}(z)$ is the Hadamard expansion defined in (2.4). Substitution of this representation into the left-hand side of (3.1) yields

$$
D w \equiv z w^{\prime \prime}+(b-z) w^{\prime}-a w=\sum_{n=0}^{\infty} \mathrm{e}^{-\lambda_{n} z} L_{n},
$$

where

$$
L_{n}=\sum_{k=0}^{\infty} a_{n k}\left\{\left\{\lambda_{n}\left(\lambda_{n}+1\right) z-b \lambda_{n}-a\right\} Y_{n k}+\left\{b-\left(1+2 \lambda_{n}\right) z\right\} Y_{n k}^{\prime}+z Y_{n k}^{\prime \prime}\right\} .
$$

We now proceed to reduce our expression for $L_{n}$ by applying, in turn, equation (2.6) to eliminate $z Y_{n k}^{\prime \prime}$ terms and (2.7) to eliminate $z Y_{n k}^{\prime}$ terms, to find

$$
\begin{aligned}
& L_{n}=\sum_{k=0}^{\infty} a_{n k}\left\{\left\{\lambda_{n}\left(\lambda_{n}+1\right) z-b \lambda_{n}-a\right.\right.\left.\left(1+2 \lambda_{n}\right)\left(\mu_{n}+k\right)\right\} Y_{n k} \\
&\left.+\left(b-\mu_{n}-k-1\right) Y_{n k}^{\prime}\right\} \\
&-\left(1+2 \lambda_{n}+\rho_{n}\right) \mathrm{e}^{-\rho_{n} z} \sum_{k=0}^{\infty} \frac{a_{n k}}{\Gamma\left(\mu_{n}+k\right)},
\end{aligned}
$$

where the coefficients $a_{n k}$ are those appearing in (2.4). Notice that the use of (2.7) has resulted in an additional series of terms involving exponentials. The remaining $Y_{n k}^{\prime}$ terms can be eliminated by using (2.5) to produce $Y$ terms with shifted index. We finally apply (2.8), which produces additional exponential terms and $Y$ terms with shifted index, to arrive at

$$
\begin{aligned}
& L_{n}=-\left(\rho_{n}+\lambda_{n}\right)\left(\rho_{n}+\lambda_{n}+1\right) \frac{\mathrm{e}^{-\rho_{n} z}}{\rho_{n}} \sum_{k=0}^{\infty} \frac{a_{n k}}{\Gamma\left(\mu_{n}+k\right)} \\
&+\sum_{k=0}^{\infty} a_{n k}\left\{\frac{\lambda_{n}\left(\lambda_{n}+1\right)}{\rho_{n}} Y_{n, k-1}+\left\{\left(\mu_{n}+k\right)\left(1+2 \lambda_{n}\right)-b \lambda_{n}-a\right\} Y_{n k}\right. \\
&\left.\quad-\rho_{n}\left(\mu_{n}+k\right)\left(b-\mu_{n}-k-1\right) Y_{n, k+1}\right\}
\end{aligned}
$$

and, after shifting indices in the sum involving the $Y_{n k}$, this becomes

$$
\begin{aligned}
& L_{n}=-\left(\rho_{n}+\lambda_{n}\right)\left(\rho_{n}+\lambda_{n}+1\right) \frac{\mathrm{e}^{-\rho_{n} z}}{\rho_{n}} \sum_{k=0}^{\infty} \frac{a_{n k}}{\Gamma\left(\mu_{n}+k\right)}+a_{n 0} \frac{\lambda_{n}\left(\lambda_{n}+1\right)}{\rho_{n}} Y_{n,-1} \\
&+\sum_{k=0}^{\infty}\left\{a_{n, k+1} \frac{\lambda_{n}\left(\lambda_{n}+1\right)}{\rho_{n}}+a_{n k}\left\{\left(\mu_{n}+k\right)\left(1+2 \lambda_{n}\right)-b \lambda_{n}-a\right\}\right. \\
&\left.\quad-a_{n, k-1} \rho_{n}\left(\mu_{n}+k-1\right)\left(b-\mu_{n}-k\right)\right\} Y_{n k},
\end{aligned}
$$

where we have defined, for notational convenience, $a_{n,-1}=0$. Note, too, the appearance of $Y_{n,-1}$ (recall (2.9)).

We can simplify our expression for $L_{n}$ by requiring that the last series in (3.4) should vanish. This is achieved by demanding that the coefficients $a_{n k}$ satisfy the 
three-term recurrence relation

$$
\begin{aligned}
a_{n, k+1} \lambda_{n}\left(\lambda_{n}+1\right)+a_{n k} \rho_{n}\left\{\left(\mu_{n}+k\right)\right. & \left.\left(1+2 \lambda_{n}\right)-b \lambda_{n}-a\right\} \\
& -a_{n, k-1} \rho_{n}^{2}\left(\mu_{n}+k-1\right)\left(b-\mu_{n}-k\right)=0
\end{aligned}
$$

for $k=0,1,2, \ldots$

\subsection{The solution $w_{1}(z)$ corresponding to $\lambda_{0}=0$}

The two linearly independent solutions of (3.1), which we denote by $w_{1}(z)$ and $w_{2}(z)$, have the leading behaviour $z^{-a}$ and $z^{a-b} \mathrm{e}^{z}$ for large $|z|$ (see [1, p. 508]). This corresponds to the choices $\lambda_{0}=0$ and $\lambda_{0}=-1$ in (3.2). We now turn to the consideration of the special cases of $L_{n}$ corresponding to these two choices of $\lambda_{0}$.

\subsubsection{The evaluation of $L_{n}, n \geqslant 0$}

Let us first consider the solution $w_{1}(z)$ with algebraic growth corresponding to $\lambda_{0}=0$. For the initial level in our Hadamard expansion of solutions of (3.1), we have $n=0$ and the expression (3.4) then reduces to

$$
L_{0}=-\left(1+\rho_{0}\right) \mathrm{e}^{-\rho_{0} z} \sum_{k=0}^{\infty} \frac{a_{0 k}}{\Gamma\left(\mu_{0}+k\right)},
$$

the other terms in the last series of (3.4) vanishing by virtue of (3.5). From the recurrence (3.5) with $\lambda_{0}=0$, we require $\mu_{0}=a$ for $a_{00} \neq 0$ and obtain the twoterm recurrence relation

$$
a_{0 k}=\frac{\rho_{0}}{k}(a+k-1)(b-a-k) a_{0, k-1} .
$$

This recurrence has the solution

$$
a_{0 k}=a_{00}\left(-\rho_{0}\right)^{k} \frac{(a)_{k}(a-b+1)_{k}}{k !}, \quad k \geqslant 0,
$$

where $(a)_{k}=\Gamma(a+k) / \Gamma(a)$ is the usual Pochhammer symbol.

In order to have incomplete gamma functions in (2.4) with positive real argument, we set $\rho_{0}=\mathrm{e}^{-\mathrm{i} \theta}$, where $\theta=\arg z$. The absolute convergence of the series $\sum a_{0 k} / \Gamma\left(\mu_{0}+k\right)$ appearing in $L_{0}$ is assured when $\left|\rho_{0}\right|<1$ by the ratio test. Provided $^{2}|\theta|<\pi$, convergence of the above series is still assured when $\left|\rho_{0}\right|=1$ if, in addition, we impose the restriction $\operatorname{Re}(b)>\operatorname{Re}(a)>0$. This results from the late behaviour of the terms in the series which, by application of the result

$$
\frac{\Gamma(k+a)}{\Gamma(k+b)} \sim k^{a-b} \quad \text { as } k \rightarrow+\infty,
$$

\footnotetext{
${ }^{2}$ We shall see in $\S 5$ that the rays $\arg z= \pm \pi$ are Stokes lines for the solution $w_{1}(z)$.
} 
is controlled by $\left(-\mathrm{e}^{-\mathrm{i} \theta}\right)^{k} k^{a-b}$. Then we have

$$
\begin{aligned}
\sum_{k=0}^{\infty} \frac{a_{0 k}}{\Gamma\left(\mu_{0}+k\right)} & =\sum_{k=0}^{\infty} \frac{a_{0 k}}{\Gamma(a+k)} \\
& =\frac{a_{00}}{\Gamma(a)} \sum_{k=0}^{\infty}(a-b+1)_{k} \frac{\left(-\rho_{0}\right)^{k}}{k !} \\
& =\frac{a_{00}}{\Gamma(a)}{ }_{1} F_{0}\left(a-b+1 ; ;-\rho_{0}\right) \\
& =\frac{a_{00}}{\Gamma(a)}\left(1+\rho_{0}\right)^{b-a-1},
\end{aligned}
$$

from which, with $\rho_{0}=\mathrm{e}^{-\mathrm{i} \theta}$, follows the final form for the zeroth level,

$$
L_{0}=-\frac{a_{00}}{\Gamma(a)} \mathrm{e}^{-|z|}\left(1+\mathrm{e}^{-\mathrm{i} \theta}\right)^{b-a} .
$$

It is this remaining contribution from the zeroth level-which we term the residual exponential that must be annihilated from the next level $n=1$. Since level $n=1$ is associated with the exponential factor $\mathrm{e}^{-\lambda_{1} z}$, it follows that, in order to produce terms in that level that can cancel the residual exponential from the zeroth level, we must set $\lambda_{1}=\rho_{0}=\mathrm{e}^{-\mathrm{i} \theta}$ when $\lambda_{0}=0$.

For the levels with $n \geqslant 1,(3.4)$ reduces to

$$
L_{n}=-\left(\rho_{n}+\lambda_{n}\right)\left(\rho_{n}+\lambda_{n}+1\right) \frac{\mathrm{e}^{-\rho_{n} z}}{\rho_{n}} \sum_{k=0}^{\infty} \frac{a_{n k}}{\Gamma\left(\mu_{n}+k\right)}+a_{n 0} \frac{\lambda_{n}\left(\lambda_{n}+1\right)}{\rho_{n}} Y_{n,-1},
$$

where, to proceed further with our analysis, we must reduce the $Y_{n,-1}$ term to a constant so that it can be used to cancel the residual exponential arising from level $n-1$. This can only be achieved by selecting $\mu_{n}=1(n \geqslant 1)$, since, in this case, the ${ }_{1} F_{1}$ function in $(2.9)$ reduces to $\mathrm{e}^{\rho_{n} z}$, yielding

$$
Y_{n,-1}(z) \equiv 1, \quad \mu_{n}=1 .
$$

Then the expression for $L_{n}$ becomes

$$
L_{n}=-\left(\rho_{n}+\lambda_{n}\right)\left(\rho_{n}+\lambda_{n}+1\right) \frac{\mathrm{e}^{-\rho_{n} z}}{\rho_{n}} \sum_{k=0}^{\infty} \frac{a_{n k}}{k !}+a_{n 0} \frac{\lambda_{n}\left(\lambda_{n}+1\right)}{\rho_{n}}, \quad n \geqslant 1,
$$

and the cancellation process can easily be achieved by a suitable choice of $a_{n 0}$ when the exponential factors in $\mathrm{e}^{-\lambda_{n} z} L_{n}$ multiplying the term proportional to $a_{n 0}$ at level $n$ and the infinite sum at level $n-1$ match, that is, when

$$
\lambda_{n+1}=\lambda_{n}+\rho_{n} .
$$

We remark that the series remaining in equation (3.10) after this cancellation process becomes the residual exponential corresponding to this level that is to be annihilated at level $n+1$.

To determine $\rho_{n}$, we examine the growth of the coefficients $a_{n k}$ defined by the recurrence (3.5) by applying dominant balance arguments outlined in [2, ch. 5]. 
From the form of the recurrence relation, it follows that the $a_{n k}$ possess the large- $k$ behaviour

$$
a_{n k} \sim A_{n} r_{1}^{k} \Gamma(k-a+1)+B_{n} r_{2}^{k} \Gamma(k+a-b+1),
$$

where $A_{n}$ and $B_{n}$ are constants independent of $k$. The quantities $r_{1}$ and $r_{2}$ are the roots of the characteristic equation

$$
\lambda_{n}\left(\lambda_{n}+1\right) r^{2}+\left(1+2 \lambda_{n}\right) \rho_{n} r+\rho_{n}^{2}=0
$$

from which $r_{1}=-\rho_{n} / \lambda_{n}$ and $r_{2}=-\rho_{n} /\left(\lambda_{n}+1\right)$. The absolute convergence of the series in (3.10) is assured if $\left|r_{1}\right|,\left|r_{2}\right|<1$. Accordingly, we define

$$
\rho_{n}=\omega_{n} \mathrm{e}^{-\mathrm{i} \theta}, \quad \omega_{n}=\min \left\{\lambda_{n}|,| \lambda_{n}+1 \mid\right\},
$$

with $\theta=\arg z$. Then, if $\omega_{n}$ is determined by the first (resp. second) choice, we have $\left|r_{2}\right|<\left|r_{1}\right|=1$ (resp. $\left|r_{1}\right|<\left|r_{2}\right|=1$ ). In view of the assignment $\rho_{0}=\mathrm{e}^{-\mathrm{i} \theta}$ at the zeroth level (where $\lambda_{0}=0$ ), we have $\omega_{0}=1$ and, from (3.11),

$$
\lambda_{n}=\Omega_{n} \mathrm{e}^{-\mathrm{i} \theta}, \quad \Omega_{n}=\sum_{r=0}^{n-1} \omega_{r}, \quad n \geqslant 1 .
$$

It follows from (3.13) and (3.14) that $\omega_{n}$ and $\Omega_{n}$ are $\theta$ dependent and that the quantities $\omega_{n}(n \geqslant 1)$ for the solution $w_{1}(z)$ are bounded away from zero, provided $|\theta|<\pi$ (see $\S 5$ for further discussion of this point).

The consideration of the convergence of the sum in (3.10) at level $n$ can be dealt with in a similar manner to that employed in the associated series at the zeroth level. The dominant late terms in the series behave like $\mathrm{e}^{\mathrm{i} k \phi_{n}} k^{-\alpha}$, where the phase $\phi_{n}$ of the larger (in modulus) of the roots $r_{1}$ and $r_{2}$ and the exponent $\alpha$ satisfy $\phi_{n}= \pm \pi$, $\alpha=a$ when $\left|r_{1}\right|=1$ or $\frac{1}{2} \pi<\left|\phi_{n}\right| \leqslant \pi, \alpha=b-a$ when $\left|r_{2}\right|=1$. Then convergence of this sum at each level again requires the conditions $\operatorname{Re}(b)>\operatorname{Re}(a)>0$ and $|\theta|<\pi$. It follows from $(2.4)$ and $(1.4 b)$ that the Hadamard sums $S_{n}(z)(n \geqslant 0)$ converge absolutely under the same restrictions.

To evaluate the sum $\sum a_{n k} / k$ !, needed to completely determine $L_{n}$ for $n \geqslant 1$, we return to the three-term recurrence (3.5) (with $\mu_{n}=1$ ) and formally multiply by $u^{k} / k$ ! and sum over $k$ from 0 to $\infty$. With

$$
G(u)=\sum_{k=0}^{\infty} a_{n k} \frac{u^{k}}{k !},
$$

we find the result expressible in the form

$$
\begin{aligned}
\lambda_{n}\left(\lambda_{n}+1\right) G^{\prime}(u)+\rho_{n} & \left(1+2 \lambda_{n}\right)(u G(u))^{\prime} \\
& -\rho_{n}\left(b \lambda_{n}+a\right) G(u)+\rho_{n}^{2}\left(u^{2} G(u)\right)^{\prime}-\rho_{n}^{2} b u G(u)=0
\end{aligned}
$$

or

$$
\frac{G^{\prime}(u)}{G(u)}=\frac{\rho_{n}(a-1)}{\rho_{n} u+\lambda_{n}}+\frac{\rho_{n}(b-a-1)}{\rho_{n} u+\lambda_{n}+1},
$$

which has the solution, using the initial value $G(0)=a_{n 0}$,

$$
G(u)=a_{n 0} \lambda_{n}^{1-a}\left(\lambda_{n}+1\right)^{1+a-b}\left(\rho_{n} u+\lambda_{n}\right)^{a-1}\left(\rho_{n} u+\lambda_{n}+1\right)^{b-a-1} .
$$


Therefore, the required sum is

$$
G(1)=\sum_{k=0}^{\infty} \frac{a_{n k}}{k !}=a_{n 0} \lambda_{n}^{1-a}\left(\lambda_{n}+1\right)^{1+a-b}\left(\rho_{n}+\lambda_{n}\right)^{a-1}\left(\rho_{n}+\lambda_{n}+1\right)^{b-a-1},
$$

giving $L_{n}(n \geqslant 1)$ the final form

$$
L_{n}=a_{n 0} \frac{\lambda_{n}\left(\lambda_{n}+1\right)}{\rho_{n}}-a_{n 0} \frac{\mathrm{e}^{-\rho_{n} z}}{\rho_{n}} \lambda_{n}^{1-a}\left(\lambda_{n}+1\right)^{1+a-b}\left(\rho_{n}+\lambda_{n}\right)^{a}\left(\rho_{n}+\lambda_{n}+1\right)^{b-a}
$$

valid provided $\operatorname{Re}(b)>\operatorname{Re}(a)>0$ when $|\theta|<\pi$.

\subsubsection{The cancellation of residual exponential terms}

The process of cancelling the residual exponential at level $n-1$ with the term $a_{n 0} \lambda_{n}\left(\lambda_{n}+1\right) / \rho_{n}$ at level $n$ can be carried out in a straightforward manner. For example, for the first level $n=1$, the term $a_{10} \lambda_{1}\left(\lambda_{1}+1\right) / \rho_{1}$ (where we recall that $\left.\lambda_{1}=\mathrm{e}^{-\mathrm{i} \theta}\right)$ in (3.16) must annihilate the residual exponential term from the zeroth level in (3.8), so

$$
a_{10} \frac{\lambda_{1}\left(\lambda_{1}+1\right)}{\rho_{1}}=-\frac{L_{0}}{\mathrm{e}^{-|z|}}=a_{00} \frac{\left(1+\mathrm{e}^{-\mathrm{i} \theta}\right)^{b-a}}{\Gamma(a)},
$$

from which it follows that

$$
a_{10}=\frac{a_{00} \rho_{1}}{\lambda_{1} \Gamma(a)}\left(1+\lambda_{1}\right)^{b-a-1} .
$$

This yields the residual exponential at level $n=1$ as

$$
-a_{00} \frac{\mathrm{e}^{-\rho_{1} z}}{\Gamma(a)} \lambda_{1}^{-a}\left(\lambda_{1}+\rho_{1}\right)^{a}\left(\lambda_{1}+\rho_{1}+1\right)^{b-a} .
$$

The first level residual term must now be cancelled with the term $a_{20} \lambda_{2}\left(\lambda_{2}+1\right) / \rho_{2}$ in $L_{2}$, so, from (3.17), we find

$$
a_{20}=a_{00} \frac{\rho_{2} \lambda_{1}^{-a}}{\Gamma(a)} \lambda_{2}^{a-1}\left(\lambda_{2}+1\right)^{b-a-1}
$$

upon setting $\lambda_{2}=\lambda_{1}+\rho_{1}$ by reason of (3.11). This produces the residual exponential term at level $n=2$ given by

$$
-a_{00} \frac{\mathrm{e}^{-\rho_{2} z}}{\Gamma(a)} \lambda_{1}^{-a}\left(\lambda_{2}+\rho_{2}\right)^{a}\left(\lambda_{2}+\rho_{2}+1\right)^{b-a},
$$

and so on. Continuing this process, we find that, at level $n \geqslant 1$,

$$
a_{n 0}=a_{00} \frac{\rho_{n} \lambda_{1}^{-a}}{\Gamma(a)} \lambda_{n}^{a-1}\left(\lambda_{n}+1\right)^{b-a-1}=\frac{a_{00} \omega_{n}}{\Gamma(a)} \Omega_{n}^{a-1}\left(1+\Omega_{n} \mathrm{e}^{-\mathrm{i} \theta}\right)^{b-a-1},
$$

with the residual exponential term given by

$$
-\frac{a_{00}}{\Gamma(a)} \mathrm{e}^{-\omega_{n}|z|} \Omega_{n+1}^{a}\left(1+\Omega_{n+1} \mathrm{e}^{-\mathrm{i} \theta}\right)^{b-a} .
$$


The other coefficients $a_{n k}$ with $n \geqslant 1, k \geqslant 1$ follow from (3.5) (where $\mu_{n}=1$ and $\left.a_{n,-1}=0\right)$ using the values of $a_{n 0}$ obtained above as starting values once a suitable choice for $a_{00}$ has been made (see below).

Thus the cancellation process can be carried out level by level to achieve the result $D w=0$ in (3.3). This generates the solution $w_{1}(z)$ of (3.1), which, from (2.4), (3.2), (3.6) and (3.14), can be expressed in the final form, valid when $\operatorname{Re}(b)>\operatorname{Re}(a)>0$ and, for $|\arg z|<\pi, z \neq 0$,

$$
w_{1}(z)=z^{-a} \sum_{k=0}^{\infty} \frac{(-)^{k}}{k ! z^{k}}(a)_{k}(a-b+1)_{k} P(a+k,|z|)+\sum_{n=1}^{\infty} \mathrm{e}^{-\Omega_{n}|z|} S_{n}^{(1)}(z),
$$

where

$$
S_{n}^{(1)}(z)=\sum_{k=0}^{\infty} \frac{a_{n k}^{(1)}}{\left(\omega_{n}|z|\right)^{k+1}} P\left(1+k, \omega_{n}|z|\right) .
$$

For convenience, we have set the coefficient $a_{00}=\mathrm{e}^{-\mathrm{i} a \theta}$ and we have introduced the superscript 1 on $S_{n}(z)$ and the coefficients $a_{n k}$ to distinguish them from the analogous quantities that will appear in the second solution $w_{2}(z)$. We note that the exponential levels $\mathrm{e}^{-\lambda_{n} z}=\mathrm{e}^{-\Omega_{n}|z|}$ appearing in the subdominant contributions of the Hadamard expansion are all real. We remark that the quantities $\omega_{n}, \Omega_{n}$ and the coefficients $a_{n k}^{(1)}$ depend on $\arg z$.

\subsection{The solution $w_{2}(z)$ corresponding to $\lambda_{0}=-1$}

We now consider the second solution $w_{2}(z)$ of (3.1) corresponding to the choice $\lambda_{0}=-1$ in (2.4) and follow the same procedure as that described for the solution $w_{1}(z)$. In this case, equation $(3.4)$ reduces to

$$
L_{0}=\left(1-\rho_{0}\right) \mathrm{e}^{-\rho_{0} z} \sum_{k=0}^{\infty} \frac{a_{0 k}}{\Gamma\left(\mu_{0}+k\right)},
$$

while the recurrence relation (3.5) shows that now we must select $\mu_{0}=b-a$ for $a_{00} \neq 0$ and

$$
a_{0 k}=a_{00} \rho_{0}^{k} \frac{(1-a)_{k}(b-a)_{k}}{k !}, \quad k \geqslant 0 .
$$

We again select $\rho_{0}=\mathrm{e}^{-\mathrm{i} \theta}$ to ensure that the incomplete gamma functions in (2.4) at the zeroth level all have positive real argument. The late terms in the series in $L_{0}$ possess the controlling behaviour $\mathrm{e}^{-\mathrm{i} k \theta} k^{-a}$, so that convergence of this series is assured if $\operatorname{Re}(b)>\operatorname{Re}(a)>0$ as before, but now in the sectors $0<|\theta| \leqslant \pi$ (recall that $\theta \equiv \arg z)$. Furthermore, we have the evaluation

$$
\sum_{k=0}^{\infty} \frac{a_{0 k}}{\Gamma(b-a+k)}=\frac{a_{00}}{\Gamma(b-a)}{ }_{1} F_{0}\left(1-a ; \mathrm{e}^{-\mathrm{i} \theta}\right)=\frac{a_{00}}{\Gamma(b-a)}\left(1-\mathrm{e}^{-\mathrm{i} \theta}\right)^{a-1}
$$

so that

$$
L_{0}=\frac{a_{00}}{\Gamma(b-a)} \mathrm{e}^{-|z|}\left(1-\mathrm{e}^{-\mathrm{i} \theta}\right)^{a}
$$

(cf. (3.7) and (3.8)). Since $L_{0}$ is multiplied by $\mathrm{e}^{-\lambda_{0} z}=\mathrm{e}^{z}$, and since this product is to be annihilated from terms arising from the next level associated with an exponential factor $\mathrm{e}^{-\lambda_{1} z}$, we must have $\mathrm{e}^{-\lambda_{0} z} \mathrm{e}^{-|z|}=\mathrm{e}^{-\lambda_{1} z}$ or $\lambda_{1}=-1+\mathrm{e}^{-\mathrm{i} \theta}$. 
For levels $n \geqslant 1$, we must again choose $\mu_{n}=1$ to ensure the term $Y_{n,-1}(z) \equiv 1$ in (3.9), with the result that (3.10) applies as before. The coefficients $a_{n k}$ are given by (3.5), but with the $\lambda_{n}$ now determined by the starting value $\lambda_{0}=-1$. The quantities $\omega_{n}, \Omega_{n}$ are given by (3.13) and (3.14) with $\rho_{n}=\omega_{n} \mathrm{e}^{-\mathrm{i} \theta}$, except that now $\lambda_{n}=-1+\Omega_{n} \mathrm{e}^{-\mathrm{i} \theta}$. The growth of the coefficients $a_{n k}$ is given by (3.12), with the roots $r_{1}$ and $r_{2}$ of the characteristic equation defined as in $\S 3.1$, so that similar arguments show that the convergence of the sums $\sum a_{n k} / \Gamma\left(\mu_{n}+k\right)$ appearing in $L_{n}$ in (3.16) requires the conditions $\operatorname{Re}(b)>\operatorname{Re}(a)>0$ and $0<|\theta| \leqslant \pi$.

The cancellation of residual exponential terms across neighbouring levels follows in a manner analogous to that described in $\S 3.1 .2$, and so we omit the details of these calculations. The residual exponential $L_{0}$ in (3.23) must annihilate the constant term in $L_{1}$ to yield

$$
a_{10}=\frac{a_{00} \omega_{1}}{\Gamma(b-a)}\left(1-\mathrm{e}^{-\mathrm{i} \theta}\right)^{a-1} .
$$

Continuation of this process produces the values $a_{n 0}$ and the residual exponential in $L_{n}$ (for $\left.n \geqslant 1\right)$ given by

$a_{n 0}=\frac{a_{00} \omega_{n}}{\Gamma(b-a)} \Omega_{n}^{b-a-1}\left(1-\Omega_{n} \mathrm{e}^{-\mathrm{i} \theta}\right)^{a-1}, \quad \frac{a_{00}}{\Gamma(b-a)} \mathrm{e}^{-\omega_{n}|z|} \Omega_{n+1}^{b-a}\left(1-\Omega_{n+1} \mathrm{e}^{-\mathrm{i} \theta}\right)^{a}$,

respectively, upon use of (3.11) (cf. (3.18) and (3.19)).

We see, therefore, that the level-by-level cancellation process in $\S 3.1 .2$ can also be carried out for the expansion corresponding to $\lambda_{0}=-1$, producing a second solution $w_{2}(z)$ satisfying (3.3). Assembling the results of this section, we see that $w_{2}(z)$ can be expressed as

$$
w_{2}(z)=\mathrm{e}^{z} z^{a-b} \sum_{k=0}^{\infty} \frac{(1-a)_{k}(b-a)_{k}}{k ! z^{k}} P(b-a+k,|z|)+\mathrm{e}^{z} \sum_{n=1}^{\infty} \mathrm{e}^{-\Omega_{n}|z|} S_{n}^{(2)}(z),
$$

where (cf. (3.21))

$$
S_{n}^{(2)}(z)=\sum_{k=0}^{\infty} \frac{a_{n k}^{(2)}}{\left(\omega_{n}|z|\right)^{k+1}} P\left(1+k, \omega_{n}|z|\right),
$$

valid when $\operatorname{Re}(b)>\operatorname{Re}(a)>0$ and $|\arg (-z)|<\pi, z \neq 0$. In a fashion akin to what we did for $w_{1}(z)$ in $(3.20)$, we have set the coefficient $a_{00}=\mathrm{e}^{\mathrm{i}(a-b) \theta}$ and we have introduced the superscript 2 on $S_{n}(z)$ and the coefficients $a_{n k}$ to distinguish them from the analogous quantities that appear in the first solution $w_{1}(z)$. We note that each of the series in (3.24) carries a factor $\mathrm{e}^{z}$, arising from the -1 term in $\lambda_{n}=-1+\Omega_{n} \mathrm{e}^{-\mathrm{i} \theta}, n \geqslant 1$, and that the remaining exponential factors $\mathrm{e}^{-\Omega_{n}|z|}$ associated with the level- $n$ expansions $S_{n}^{(2)}(z)$ are all real exponentially decaying terms. The quantities $\omega_{n}$ and $\Omega_{n}$ appearing in both (3.20) and (3.24) are the same.

To conclude this section, we remark that if, instead of the choice $\rho_{0}=\mathrm{e}^{-\mathrm{i} \theta}$ in (3.22), we put $\rho_{0}=1$, then $L_{0} \equiv 0$ and the expansion procedure terminates at the zeroth level. We then obtain the solution consisting of a single Hadamard expansion that involves incomplete gamma functions with complex argument when 
$\theta \neq 0$ given by

$$
a_{00} z^{a-b} \mathrm{e}^{z} \sum_{k=0}^{\infty} \frac{(1-a)_{k}(b-a)_{k}}{k ! z^{k}} P(b-a+k, z) .
$$

However, this representation lacks the separation into levels with exponentially decaying factors and incomplete gamma functions of real argument. In [5], this solution is shown (when $a_{00}=1$ ) to be a representation of the confluent hypergeometric function $M(a, b, z)$.

\subsection{Matching}

Before moving to a numerical illustration, we address the matter of identifying our Hadamard expansion solutions $w_{1}(z)$ and $w_{2}(z)$, defined in (3.20) and (3.24), in terms of the familiar confluent hypergeometric functions $M(a, b, z)={ }_{1} F_{1}(a ; b ; z)$ and $U(a, b, z)$. It is clear that $w_{1}(z)$ and $w_{2}(z)$ must be expressible as linear combinations of these two solutions in the form

$$
\begin{aligned}
U(a, b, z) & =C_{1} w_{1}(z)+D_{1} w_{2}(z), \\
M(a, b, z) & =C_{2} w_{1}(z)+D_{2} w_{2}(z),
\end{aligned}
$$

where the $C_{j}, D_{j}(j=1,2)$ are constants and $z$ lies in the principal sheet.

The confluent hypergeometric functions have the leading asymptotic behaviour for large $|z|$ given by

$$
\begin{aligned}
\frac{M(a, b, z)}{\Gamma(b)}=\frac{\mathrm{e}^{\pi \mathrm{i} a} z^{-a}}{\Gamma(b-a)}\left(1+\mathcal{O}\left(z^{-1}\right)\right)+\frac{\mathrm{e}^{z} z^{a-b}}{\Gamma(a)}\left(1+\mathcal{O}\left(z^{-1}\right)\right), & & -\frac{1}{2} \pi<\arg z<\frac{3}{2} \pi \\
U(a, b, z)=z^{-a}\left(1+\mathcal{O}\left(z^{-1}\right)\right), & & -\frac{3}{2} \pi<\arg z<\frac{3}{2} \pi
\end{aligned}
$$

(see $\left[1\right.$, eqns (13.5.1) and (13.5.2)]). The Poincaré asymptotic expansions of $w_{1}(z)$ and $w_{2}(z)$ follow from $(3.20)$ and $(3.24)$ by formal replacement of the incomplete gamma functions with unity in the Hadamard expansions at the zeroth level (see [7] for a justification of this process), to find

$$
\begin{array}{ll}
w_{1}(z) \sim z^{-a} \sum_{k=0}^{\infty} \frac{(-)^{k}(a)_{k}(a-b+1)_{k}}{k ! z^{k}}, & |\arg z|<\pi, \\
w_{2}(z) \sim z^{a-b} \mathrm{e}^{z} \sum_{k=0}^{\infty} \frac{(1-a)_{k}(b-a)_{k}}{k ! z^{k}}, & |\arg (-z)|<\pi .
\end{array}
$$

Comparison with the above leading behaviours shows that $C_{1}=1, D_{1}=0$, to yield

$$
U(a, b, z)=w_{1}(z), \quad|\arg z|<\pi
$$

Similarly for the function $M(a, b, z)$, the solution ${ }^{3} w_{2}(z)$ is exponentially large along a ray in the right-hand plane, while along a ray in $\frac{1}{2} \pi<|\arg z|<\pi$ the

\footnotetext{
${ }^{3}$ We remark that the solution $w_{2}(z)$ is a constant multiple of the solution $V(a, b, z)$ defined in $[4$, p. 256].
} 
solution $w_{1}(z)$ is dominant. Hence $C_{2}=\Gamma(b) / \Gamma(a)$ and $D_{2}=\mathrm{e}^{\pi \mathrm{i} a} \Gamma(b) / \Gamma(b-a)$, to yield

$$
M(a, b, z)=\frac{\Gamma(b)}{\Gamma(a)} w_{1}(z)+\mathrm{e}^{\pi \mathrm{i} a} \frac{\Gamma(b)}{\Gamma(b-a)} w_{2}(z), \quad|\arg (-z)|<\pi .
$$

These expansions were obtained in [6] by a process derived from the Laplace integral representations for the confluent hypergeometric functions.

\section{Modified Hadamard expansions}

In using our expansions to generate numerical approximations of $U(a, b, z)$ and $M(a, b, z)$, we must deal with the slow convergence of the tails of the Hadamard expansions mentioned in $\S 1$ (also see [5]). Thus, at each level $n$, we consider a typical Hadamard expansion $S_{n}(z)$ in (2.4) and write

$$
S_{n}(z)=\sum_{k=0}^{M_{n}-1} a_{n k} Y_{n k}(z)+T_{n}\left(M_{n} ; z\right),
$$

where $M_{n}$ is (for the moment) an arbitrary truncation index and the tail is

$$
T_{n}\left(M_{n} ; z\right)=\sum_{k=M_{n}}^{\infty} a_{n k} Y_{n k}(z) .
$$

As has been done throughout in $[5,6]$, we employ the strategy of expressing the $Y_{n k}$ in (4.2) in terms of the function ${ }_{1} F_{1}$ by means of

$$
P(a, x)=\frac{x^{a} \mathrm{e}^{-x}}{\Gamma(a+1)}{ }_{1} F_{1}(1 ; 1+a ; x)
$$

(recall (1.4)), followed by use of the Maclaurin series for ${ }_{1} F_{1}$ and an interchange in the order of summation. Proceeding in this manner for the zeroth-level tail for $w_{1}(z)$ (with $\lambda_{0}=0$ and $a_{00}=\mathrm{e}^{-\mathrm{i} a \theta}$ ), we find

$$
\begin{aligned}
T_{0}\left(M_{0} ; z\right) & =\mathrm{e}^{-|z|} \sum_{r=0}^{\infty}|z|^{r} \sum_{k=M_{0}}^{\infty} \frac{(-)^{k}(a)_{k}(a-b+1)_{k}}{k ! \Gamma(a+k+r+1)} \mathrm{e}^{-\mathrm{i}(a+k) \theta} \\
& =\mathrm{e}^{-|z|-\mathrm{i} a \theta} \sum_{r=0}^{\infty} \sigma_{r 0}\left(M_{0}\right) \chi_{0}^{r},
\end{aligned}
$$

where $\chi_{0}=|z| / M_{0}$ and the coefficients $\sigma_{r 0}$ are defined by

$$
\begin{aligned}
\sigma_{r 0}\left(M_{0}\right) & =M_{0}^{r} \sum_{k=M_{0}}^{\infty} \frac{(a)_{k}(a-b+1)_{k}\left(-\mathrm{e}^{-\mathrm{i} \theta}\right)^{k}}{k ! \Gamma(a+k+r+1)} \\
& \left.=\frac{M_{0}^{r}}{\Gamma(a+r+1)}{ }_{2} F_{1}\left(a, a-b+1 ; a+r+1 ;-\mathrm{e}^{-\mathrm{i} \theta}\right)\right\rfloor M_{0} .
\end{aligned}
$$

Here, ${ }_{2} F_{1}(\cdot)_{\rfloor} M_{0}$ denotes a truncated ${ }_{2} F_{1}$ function; that is, the function ${ }_{2} F_{1}$ with the first $M_{0}$ terms of its Maclaurin series deleted. 
A similar process applies to the tails produced by truncating the series at other levels. For $n \geqslant 1$, equation (4.2) becomes

$$
T_{n}\left(M_{n} ; z\right)=\sum_{k=M_{n}}^{\infty} \frac{a_{n k}}{\left(\omega_{n}|z|\right)^{k+1}} P\left(1+k, \omega_{n}|z|\right),
$$

which, after using (1.4) and the subsequent representation of ${ }_{1} F_{1}$ as a Maclaurin series and a change in the order of summation, becomes

$$
\begin{aligned}
T_{n}\left(M_{n} ; z\right) & =\mathrm{e}^{-\omega_{n}|z|} \sum_{r=0}^{\infty}\left(\omega_{n}|z|\right)^{r} \sum_{k=M_{n}}^{\infty} \frac{a_{n k}}{(k+1) !} \frac{1}{(k+2)_{r}} \\
& =\mathrm{e}^{-\omega_{n}|z|} \sum_{r=0}^{\infty} \sigma_{r n}\left(M_{n}\right) \chi_{n}^{r},
\end{aligned}
$$

with $\chi_{n}=\omega_{n}|z| / M_{n}$ and

$$
\sigma_{r n}\left(M_{n}\right)=M_{n}^{r} \sum_{k=M_{n}}^{\infty} \frac{a_{n k}}{(k+r+1) !}
$$

in view of the identity $(k+1) !(k+2)_{r}=(k+r+1)$ !.

An integral representation of $\sigma_{r n}$ for $n \geqslant 1$ can be determined from the generating function for the coefficients $a_{n k}$. With $G_{n} \equiv G$ in $(3.15)$, we obtain (see $[5, \S 5]$ )

$$
\frac{1}{r !} \int_{0}^{1}(1-u)^{r} G_{n}(u) \mathrm{d} u=\sum_{k=0}^{\infty} \frac{a_{n k}}{(r+k+1) !}
$$

whence, in view of (4.5), we deduce that

$$
\sigma_{r n}\left(M_{n}\right)=M_{n}^{r}\left\{\frac{1}{r !} \int_{0}^{1}(1-u)^{r} G_{n}(u) \mathrm{d} u-\sum_{k=0}^{M_{n}-1} \frac{a_{n k}}{(r+k+1) !}\right\} .
$$

This expression is suitable for efficient machine-assisted computation, and avoids resorting to infinite series to effect the computation of the $\sigma_{r n}$.

\section{A numerical example}

The results of the preceding sections can be assembled to compute an effective high-precision approximation scheme for the confluent hypergeometric functions $U(a, b, z)$ and $M(a, b, z)$. We illustrate the procedure with one example concerning the computation of $U(a, b, z)$; other examples, including the Bessel functions, the incomplete Airy function and the gamma function, can be found in [5-7]. From (3.27) and (3.20), we have

$$
U(a, b, z)=z^{-a} \sum_{k=0}^{\infty} \frac{(-)^{k}}{k ! z^{k}}(a)_{k}(a-b+1)_{k} P(a+k,|z|)+\sum_{n=1}^{\infty} \mathrm{e}^{-\Omega_{n}|z|} S_{n}^{(1)}(z),
$$

where $S_{n}^{(1)}(z)$ is defined in (3.21). The coefficients $a_{n k}^{(1)}$ appearing in $S_{n}^{(1)}(z)$ are defined by means of the recurrence relation (3.5) with $\mu_{n}=1, \rho_{n}=\omega_{n} \mathrm{e}^{-\mathrm{i} \theta}, \lambda_{n}=$ 


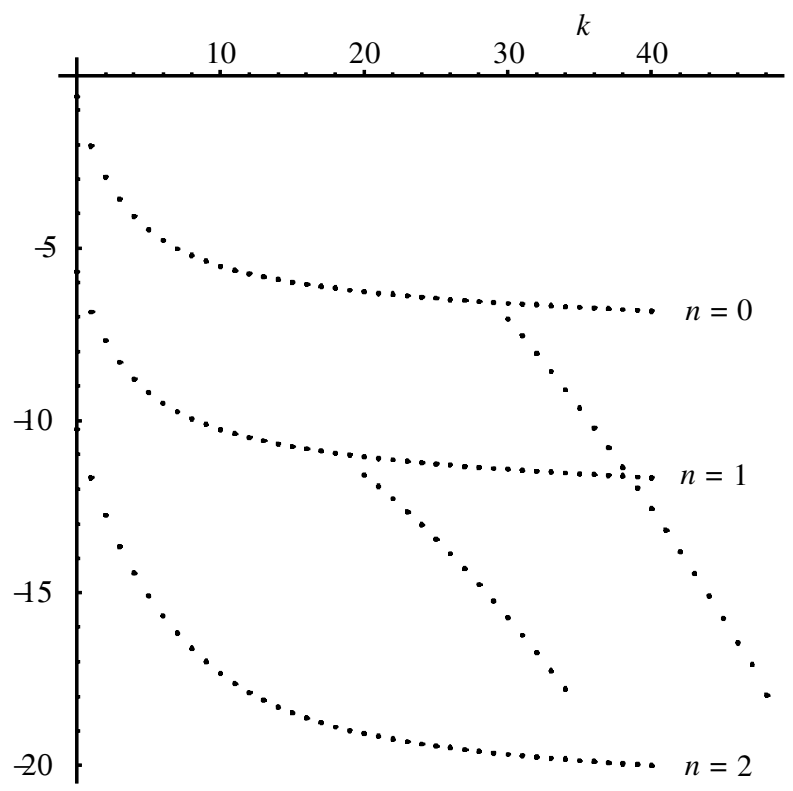

Figure 2. Magnitudes of terms (plotted on a $\log _{10}$ scale) against ordinal number $k$ in levels 0,1 and 2 of the Hadamard expansion of $U\left(\frac{3}{5}, 1, x\right)$, and of terms in the rearranged tails resulting from the choices $M_{0}=30$ and $M_{1}=20$. The first term in the third level (not shown) is of order $10^{-20}$.

$\Omega_{n} \mathrm{e}^{-\mathrm{i} \theta}$ and the choice $a_{00}=\mathrm{e}^{-\mathrm{i} a \theta}$. From this, it is evident that these coefficients are $\theta$ dependent. The quantities $\omega_{n}$ and $\Omega_{n}$ are defined in (3.13) and (3.14) and are also seen to be $\theta$ dependent. For the solution $w_{1}(z)$ at level $n=1$, we have $\omega_{1}=\min \left\{1,\left|1+\mathrm{e}^{-\mathrm{i} \theta}\right|\right\}$ (since $\lambda_{1}=\mathrm{e}^{-\mathrm{i} \theta}$ ), that is,

$$
\omega_{1}= \begin{cases}1, & |\theta| \leqslant \frac{2}{3} \pi, \\ (2(1+\cos \theta))^{1 / 2}, & \frac{2}{3} \pi \leqslant|\theta|<\pi .\end{cases}
$$

In the sector $|\arg z| \leqslant \frac{1}{2} \pi$, the $\omega_{n}$ for the solution $w_{1}(z)$ are consequently independent of $\theta$ and we have $\omega_{n}=\Omega_{n}=2^{n-1}(n \geqslant 1)$. In the sector $\frac{1}{2} \pi<|\arg z|<\pi$, however, an increasingly finely structured piecewise $\theta$ dependence holds for the $\omega_{n}$ at the higher levels $n \geqslant 1$, of which (5.2) represents this dependence at level $n=1$ (for a further discussion of this point, see [6]).

The Hadamard series in (5.1) are written in the modified form (4.1) and (4.2), where the truncation indices $M_{n}$ of the main sums and $N_{n}$ of the rearranged absolutely convergent tails $T_{n}\left(M_{n} ; z\right)$ are chosen according to the level of precision sought. For example, if we wished to know the value of $U\left(\frac{3}{5}, 1, z\right)$ for $z=10$ to an accuracy of at least $10^{-20}$, say, then we must examine the magnitudes of the terms present in the series for the truncated Hadamard series and their associated tails. This can be done once initial truncation indices for the various levels have been determined.

In order to ensure a rapid decay of the terms in the rearranged tails $T_{n}\left(M_{n} ; z\right)$, we will want to insist on the variable $\chi_{n}$ in (4.3) and (4.4) satisfying $\chi_{n}<1$, which in turn requires, for the zeroth level, that $M_{0} \geqslant\lfloor|z|\rfloor+1$, and for levels $n \geqslant 1$, 
Table 1. Absolute values of the error in $U(a, b, z)$ when $a=\frac{3}{5}, b=1$

and $z=10$ at different levels $n$

(The truncation indices employed are indicated in the text.)

\begin{tabular}{ccc}
\hline$n$ & approximation to $U(a, b, 10)$ & $\mid$ error $\mid$ \\
\hline 0 & 0.24310114584288995727 & $1.888 \times 10^{-6}$ \\
1 & 0.24310303349194502562 & $5.225 \times 10^{-11}$ \\
2 & $0.243103033544192235 \mathbf{7 5}$ & $6.954 \times 10^{-20}$ \\
exact & 0.24310303354419223582 & 0 \\
\hline
\end{tabular}

Table 2. Absolute values of the error committed in approximating $U\left(\frac{3}{5}, 1,10 \mathrm{e}^{\mathrm{i} \theta}\right)$ for different $\theta$

\begin{tabular}{cc}
\hline$\theta$ & $\mid$ error $\mid$ \\
\hline 0 & $6.954 \times 10^{-20}$ \\
$\frac{1}{6} \pi$ & $6.695 \times 10^{-20}$ \\
$\frac{1}{3} \pi$ & $6.883 \times 10^{-20}$ \\
$\frac{1}{2} \pi$ & $7.212 \times 10^{-20}$ \\
\hline
\end{tabular}

that $M_{n} \geqslant\left\lfloor\left|\omega_{n} z\right|\right\rfloor+1$. Once the $M_{n}$ have been selected, the terms in the tails can be computed by truncating them after $N_{n}$ terms commensurate with the level of precision required. We show in figure 2 the magnitude of the terms (on a logarithmic scale) in the different levels of the expansion (5.1) against ordinal number for $z=10$ and a given choice of truncation indices. We observe that the terms in the tails of the modified expansions decay at a rate that is roughly speaking the same as that in the initial asymptotic-like phase of the corresponding finite main sums.

With these considerations in mind, we make the choices $\left(M_{0}, N_{0}\right)=(30,22)$, $\left(M_{1}, N_{1}\right)=(20,18)$ and $\left(M_{2}, N_{2}\right)=(40,0)$ (that is, no terms from the tail at level 2 are used) to compute our estimated value of $U\left(\frac{3}{5}, 1,10\right)$ from $(5.1)$ to an accuracy of order $10^{-20}$. The results of these computations using MATHEMATICA are presented in table 1.

The Hadamard expansion (5.1) is quite robust in the sense that it will produce high-quality estimates for $U(a, b, z)$ over a wide range of values of the phase of $z$ using truncation indices selected for a fixed phase of $z$. To illustrate this, with our truncation indices chosen for $z=10$ (that is, $\arg z=0$ ), we obtain estimates for $U\left(\frac{3}{5}, 1,10 \mathrm{e}^{\mathrm{i} \theta}\right)$ of a calibre comparable to that obtained for the case with $\theta=0$ in the whole right half-plane. A summary of the accuracy is supplied in table 2 .

As the phase varies so that $z$ swings round towards the negative real axis, the expansion (5.1) retains its validity although the exponential separation between the different levels $n \geqslant 1$ progressively decreases on account of the fact that $\omega_{n} \rightarrow 0$ (and $\Omega_{n} \rightarrow 1$ ) as $|\theta| \rightarrow \pi$ (cf. (5.2)). This is how the Stokes phenomenon manifests itself in a Hadamard expansion: the rays arg $z= \pm \pi$ are Stokes lines for the function $U(a, b, z)$. As a Stokes line is approached, the different Hadamard expansions in (5.1) begin to 'sense' this approach at a maximum angular distance of $\frac{1}{2} \pi$ on either side of a Stokes line (see [6]) by progressively losing their exponential separation. 
Consequently, a given desired level of precision in an estimate produced by (5.1) will require the use of more levels, and more terms in the tails as $\theta \rightarrow \pm \pi$. A better approach is to use connection formulae to render evaluation in the left half-plane in terms of other functions, better suited for computation there.

An analogous situation occurs for $M(a, b, z)$ in the neighbourhood of the ray $\arg z=0$, which is a Stokes line for this function. For the solution $w_{2}(z)$, the sectorial structure of the quantities $\omega_{n}$ and $\Omega_{n}$ is reversed, that is, these quantities are independent of $\theta$ in the sectors $\frac{1}{2} \pi \leqslant|\theta| \leqslant \pi$ and possess a similar $\theta$ dependence to that for the solution $w_{1}(z)$ in the sector $|\theta|<\frac{1}{2} \pi$, with the result that $\omega_{n} \rightarrow 0$ (and $\Omega_{n} \rightarrow 1$ ) as $\theta \rightarrow 0$ for $n \geqslant 1$. We remark that the matter of handling the Stokes phenomenon has not been dealt with within the confines of the differential equations theory described in the present paper. The reader interested in the Stokes phenomenon can find a detailed account in [7], developed in the setting of Hadamard expansions derived from Laplace integral representations.

\section{Closing remarks}

On the strength of the technique outlined in the development of Hadamard expansions of solutions of the confluent hypergeometric equation, it is fair to say that the elements of a manageable theory of developing such expansions directly from differential equations are now in place. The special properties of the $Y_{n k}$ listed in lemma 2.1 are, however, tightly coupled to differential equations of hypergeometric type (especially in view of (2.6)). We mention here that preliminary investigations suggest additional classes of hypergeometric differential equations may be within the scope of the methods presented in this paper.

To give the reader an idea of how the method changes if the differential equation under analysis is perturbed from the form discussed here, let us suppose we alter Kummer's equation (3.1) to

$$
z w^{\prime \prime}+\left(b-z+\frac{c}{z}\right) w^{\prime}-\left(a+\frac{d}{z}\right) w=0 .
$$

This perturbs each of the derived and underived terms in (3.1) at a singularity of the differential equation.

The reduction process can be carried out as in $\S 3$, with some minor modification. Properties supplied in lemma 2.1 apply as before, but now need extension to handle additional forms appearing in the reduction process for each $L_{n}$ leading to the formulation of the recurrence relation for the coefficients $a_{n k}$ and the ensuing residual exponential terms. Additionally, it may be necessary to modify the scale $\left\{Y_{n k}\right\}$ used in fashioning the Hadamard expansion.

For example, for (6.1), terms such as $z^{-1} Y_{n k}, z^{-2} Y_{n k}$ and $z^{-3} Y_{n k}$ arise early on in the reduction process for the levels $L_{n}$. To cope with these, it is convenient to extend the list of relations in lemma 2.1 to include

$$
\begin{aligned}
\frac{1}{z} Y_{n k} & =\rho_{n} Y_{n, k+1}+\frac{\mathrm{e}^{-\rho_{n} z}}{z \Gamma\left(\mu_{n}+k+1\right)}, \\
\frac{1}{z^{2}} Y_{n k} & =\rho_{n}^{2} Y_{n, k+2}+\left(1+\frac{\rho_{n} z}{\mu_{n}+k+1}\right) \frac{\mathrm{e}^{-\rho_{n} z}}{z^{2} \Gamma\left(\mu_{n}+k+1\right)},
\end{aligned}
$$




$$
\begin{array}{r}
\frac{1}{z^{3}} Y_{n k}=\rho_{n}^{3} Y_{n, k+3}+\left(1+\frac{\rho_{n} z}{\mu_{n}+k+1}+\frac{\left(\rho_{n} z\right)^{2}}{\left(\mu_{n}+k+2\right)\left(\mu_{n}+k+1\right)}\right) \\
\times \frac{\mathrm{e}^{-\rho_{n} z}}{z^{3} \Gamma\left(\mu_{n}+k+1\right)}
\end{array}
$$

these extensions all arise upon repeated application of (2.8). It is an immediate consequence of these properties that the residual exponential terms will also include factors of $1 / z, 1 / z^{2}$ and $1 / z^{3}$, making the cancellation process from one level to the next a more delicate affair as all coefficients of these reciprocal powers of $z$ must be matched simultaneously.

In allowing the perturbations we consider here, it is natural to consider modifications of the $Y_{n k}$, so that, instead of seeking an expansion of the form in (2.3) and (2.4), we can consider other possibilities such as

$$
w(z)=\sum_{n=0}^{\infty} \mathrm{e}^{-\lambda_{n} z} \tilde{S}_{n}(z), \quad \text { with } \tilde{S}_{n}(z)=\sum_{k=0}^{\infty} a_{n k} Y_{n k}(z)\left(1+\frac{A_{n k}}{\rho_{n} z}\right) .
$$

The additional factor $1+A_{n k}\left(\rho_{n} z\right)^{-1}$ appended to $Y_{n k}$ may facilitate the cancellation process.

These variations lead to more complex recurrences and residual exponentials. For (6.1), the $a_{n k}$ counterpart to (3.5) includes at least one additional term if $c \neq 0$ in (6.1), with $d$ making its presence felt in the third term of the perturbed counterpart to (3.5). Use of a modified scale $\left\{Y_{n k}(z) \cdot\left(1+A_{n k}\left(\rho_{n} z\right)^{-1}\right)\right\}$ results in a similarly laden recurrence in $a_{n k}$, which is simultaneously a recurrence in $A_{n k}$ and includes yet another term,

$$
\begin{aligned}
& a_{n, k+1} \lambda_{n}\left(\lambda_{n}+1\right) \\
& +a_{n k} \rho_{n}\left\{\left(\mu_{n}+k\right)\left(1+2 \lambda_{n}\right)-b \lambda_{n}-a+\frac{\lambda_{n}\left(\lambda_{n}+1\right)}{\rho_{n}} A_{n k}\right\} \\
& +a_{n, k-1} \rho_{n}^{2}\left\{\left[1+(2-b) \lambda_{n}-a+\left(\mu_{n}+k-1\right)\left(1+2 \lambda_{n}\right)\right] \frac{A_{n, k-1}}{\rho_{n}}\right. \\
& \left.-\left[\lambda_{n} c+d+\left(\mu_{n}+k-1\right)\left(b-\mu_{n}-k\right)\right]\right\} \\
& +a_{n, k-2} \rho_{n}^{3}\left\{( 2 - b - d - \lambda _ { n } c ) \frac { A _ { n , k - 2 } } { \rho _ { n } } \left[\begin{array}{c}
\left.\left.\mu_{n}+k-2\right)\left[c+\left(b-\mu_{n}-k-1\right) \frac{A_{n, k-2}}{\rho_{n}}\right]\right\} \\
\rho_{n}
\end{array}\right.\right.
\end{aligned}
$$

We observe that, with $c=d=0$ and $A_{n k}=0$ for all $n$ and $k$, this five-term recurrence relation reduces to (3.5). The solution of such a double recurrence is problematical, at best.

Other types of differential equations may prove more amenable to the techniques described in this paper. 


\section{Acknowledgments}

D.K. gratefully acknowledges the hospitality of the University of Abertay Dundee, where he was a visitor during this research, and the Natural Sciences and Engineering Research Council of Canada for underwriting a portion of the costs of this research visit.

\section{References}

1 M. Abramowitz and I. Stegun (eds). Handbook of mathematical functions (New York: Dover, 1965).

2 C. M. Bender and S. A. Orszag. Advanced mathematical methods for scientists and engineers (McGraw-Hill, 1978).

3 W. F. Kibble. A Bessel function expansion in terms of incomplete gamma functions. J. Indian Math. Soc. 3 (1939), 271-294.

4 F. W. J. Olver. Asymptotics and special functions (Academic, 1974).

5 R. B. Paris. On the use of Hadamard expansions in hyperasymptotic evaluation. I. Real variables. Proc. R. Soc. Lond. A 457 (2001), 2835-2853.

6 R. B. Paris. On the use of Hadamard expansions in hyperasymptotic evaluation. II. Complex variables. Proc. R. Soc. Lond. A 457 (2001), 2855-2869.

7 R. B. Paris. On the use of Hadamard expansions in hyperasymptotic evaluation: Laplacetype integrals. J. Comp. Appl. Math. (In the press.)

8 G. N. Watson. A treatise on the theory of Bessel functions (Cambridge University Press, $1952)$.

( Issued 27 February 2004) 